\section{IMR goes Online}

Der Intensivkurs Muskuloskelettale Radiologie (IMR) fand dieses Jahr als kompaktes Online-Format eingegliedert in den RÖKO DIGITAL statt. Am 5. September $\mathbf{2 0 2 0}$ folgten knapp 800 Teilnehmerinnen und Teilnehmer den Vorträgen der hochkarätigen internationalen Referenten in drei wissenschaftlichen Sessions.

Themenschwerpunkte der IMR-Spezialausgabe waren die Schulter- und Kniediagnostik. Unter der wissenschaftlichen Leitung von Prof. Dr. Marc Regier konnte erstmals ein Top-Referent aus den USA für einen Vortrag gewonnen werden. Prof. Bill Palmer vom Massachusetts General Hospital Harvard/Boston sorgte mit seinem Vortrag
„Acute and chronic ACL tears and secondary sign“ für Begeisterung bei den Teilnehmerinnen und Teilnehmern.

Sollten Sie die spannenden Vorträge des Intensivkurses Muskuloskelettale Radiologie verpasst haben, können Sie sich die Vorträge (die Anmeldung zum RÖKO DIGITAL vorausgesetzt) noch bis Ende Januar 2021 auf conrad, der digitalen Lernplattform der DRG, anschauen.

\section{IMR 2021}

Im kommenden Jahr wird der IMR in Würzburg aufgrund der durch COVID-19 bedingten unklaren Planungssituation pausieren.
Wir hoffen aber, Sie vom 10. bis 11. September 2021 wieder wie gewohnt zum IMR in der Bucerius Law School in Hamburg begrüßen zu können.

\section{SAVE THE DATE}

10.-11. September 2021, IMR Hamburg

Mehr Informationen:

www.drg.de > IMR 2021 